\title{
Research and publication ethics of the Journal of Exercise Rehabilitation
}

\author{
Chang-Ju Kim (i) https://orcid.org/0000-0003-4749-5795
}

Many articles from many countries have been submitted to the Journal of Exercise Rebabilitation (JER). In this issue, the studies on the various effects of exercise, such as antioxidant effects, the effects of exercise on blood sugar, hypertension, and also on anxiety are published. Studies on the effect of rehabilitation training in an artificially heated environment and the effect of whole body vibration are also included in this issue.

The editorial board of the JER strengthened the research ethics and publication ethics to the submitted articles. In addition to being approved by the ethics committee, a detailed explanation of the experiment needs to be given to the participants. The editorial board of JER also measured the similarity of all submitted manuscripts. The high degree of similarity with other studies is one of the important factors rejected by the JER. Compliance with research ethics and publication ethics is a fundamental condition for the JER to maintain its status as an international journal.

The editorial board of the JER also attaches importance to the format of the submitted manuscripts. It is important to comply with the JER submission guidelines by referring to "Instructions to Authors" or existing published articles.

It is an important role of the JER to inform the effects of exercise in various disease states. However, in order to expand the scope and role of exercise rehabilitation, mechanisms of exercise for rehabilitation should be identified. Evaluating the mechanisms of exercise rehabilitation is a necessary task for the development of a new therapeutic area of exercise rehabilitation. JER welcomes the studies on the mechanisms of exercise rehabilitation.

The editorial board of the JER has been striving to become an international journal in the section of exercise rehabilitation since 2013, and JER has made a lot of progress in terms of content. Now we look forward to further development of JER as an international journal.

\section{Conflict of Interest}

No potential conflict of interest relevant to this article was reported.

President of the Korean Society of Exercise Rehabilitation Department of Physiology, College of Medicine, Kyung Hee University 26 Kyungheedae-ro, Dongdaemun-gu, Seoul 02447, Korea E-mail: changju@khu.ac.kr 